\title{
Primary Hyperparathyroidism: Can Parathyroid Carcinoma Be Anticipated on Clinical and Biochemical Grounds? Report of Nine Cases and Review of the Literature
}

\author{
John H. Robert, MD, ${ }^{1}$ Andrea Trombetti, MD, ${ }^{2}$ Alain Garcia, MD, ${ }^{1}$ \\ Jean-Claude Pache, MD ${ }^{3}$ François Herrmann, MD, MPH, ${ }^{4}$ Anastase Spiliopoulos, MD,${ }^{1}$ and \\ René Rizzoli, $\mathrm{MD}^{2}$
}

\footnotetext{
${ }^{1}$ Department of Surgery, Service of Thoracic Surgery, Geneva University Hospitals, CH-1211 Geneva 14, Switzerland ${ }^{2}$ Service of Bone Diseases, World Health Organization Collaborating Center for Osteoporosis Prevention, Geneva University Hospitals, CH-1211 Geneva 14, Switzerland

${ }^{3}$ Department of Pathology, Division of Clinical Pathology, Geneva University Hospitals, CH-1211 Geneva 14, Switzerland

${ }^{4}$ Department of Rehabilitation and Geriatrics, Geneva University Hospitals, CH-1211 Geneva 14, Switzerland
}

Primary hyperparathyroidism (PHP) is caused in the vast majority of cases by parathyroid adenomas or hyperplasias, but occasionally it is caused by

Received June 1, 2004; accepted January 19, 2005; published online

Address correspondence and reprint requests to: John H. Robert, MD; E-mail: john.h.robert@ hcuge.ch.

Published by Springer Science+Business Media, Inc. @ 2005 The Society of Surgical Oncology, Inc. parathyroid carcinomas (PC). In all three instances, the clinical and biological picture may be strikingly similar. ${ }^{1}$ Because of the lack of specific clinical features and because percutaneous aspiration biopsies are rightly contraindicated, ${ }^{2}$ a diagnosis of $\mathrm{PC}$ is difficult to establish ${ }^{3}$ and is seldom available at the time of the first operation. ${ }^{1,49}$ The similarity in clinical presentation is unfortunate, because PC may be plagued by recurrences - one of the hallmarks of this tenacious tumor ${ }^{10,1}$ - and because recurrences sel- 


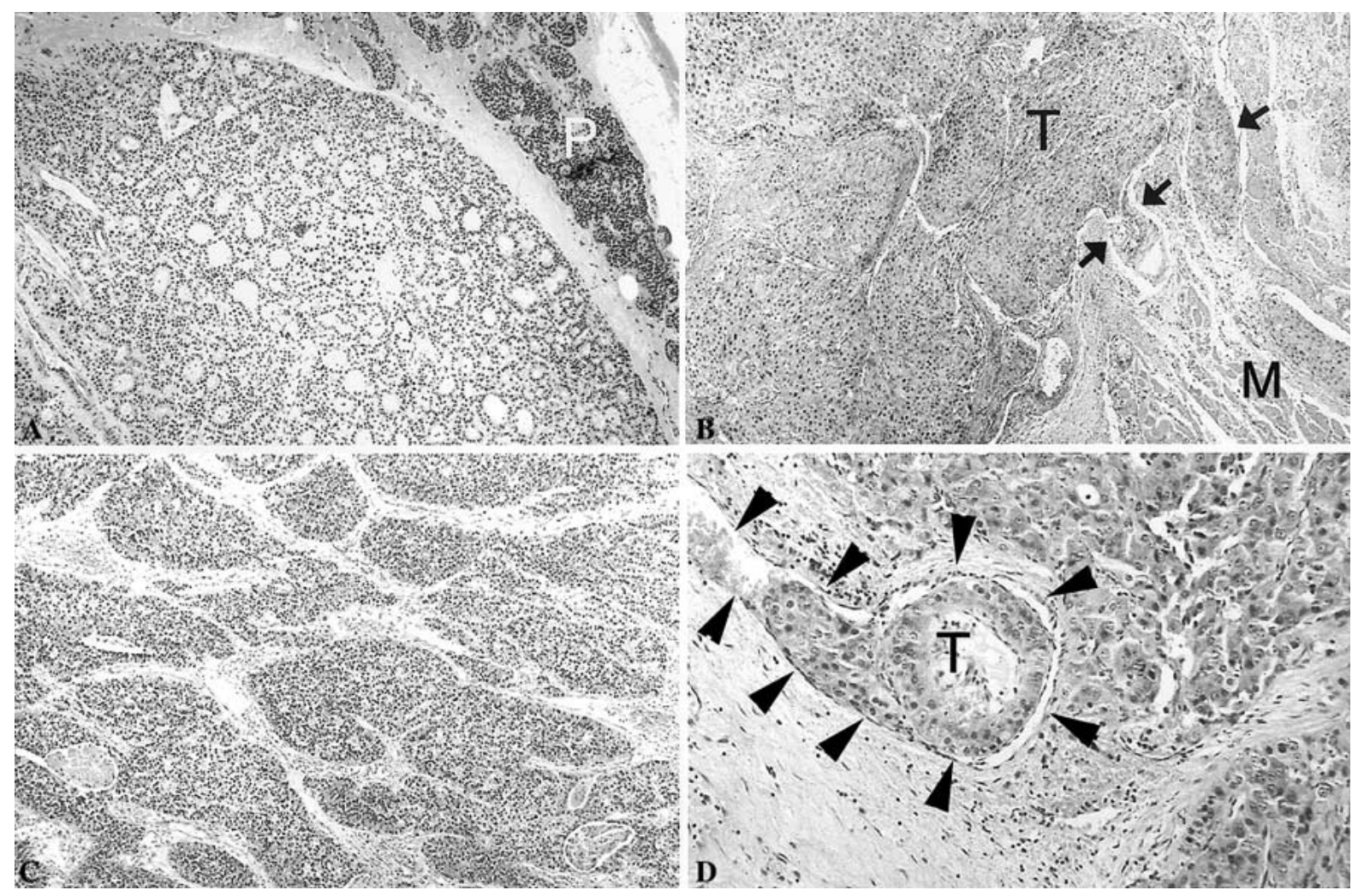

FIG. 1. Histological appearance of parathyroid adenoma (A) and carcinoma (B-D). (A) Adenoma is lined by a capsule, and nontumorous glandular parenchyma is compressed (upper right corner; stain, hematoxylin and eosin; original magnification, $\times 100)$. (B) Parathyroid carcinoma: tumor (T) invades the capsule (black arrows). M, muscle (stain, hematoxylin and eosin; original magnification, $\times 20$ ). (C) Carcinomatous cells are separated by thick fibrous bands (stain, hematoxylin and eosin; original magnification, $\times 100$ ). (D) Tumor invasion (T) is seen within a blood vessel (arrowheads; stain, hematoxylin and eosin; original magnification, $\times 200$ ).

dom lend themselves to curative therapies, be they surgical or conservative. ${ }^{2,6,11-14}$

Recurrences are frequently cervical (up to two thirds of cases ${ }^{4}$ and usually within 3 years ${ }^{11}$ ), and one wonders whether some of these cases could have been prevented had the diagnosis of PC been ascertained and, accordingly, more generous excisions had been performed at the time of the first operation (for instance, on the contiguous thyroid lobe ${ }^{12}$ or on enlarged nodes ${ }^{15}$ ).

The purpose of this study was to reevaluate the clinical and biochemical parameters that have been used to differentiate PC from benign PHP at the time of the first operation. To achieve this, patients with benign PHP were compared retrospectively with patients with PC who underwent operation in the same department during the same period of time. Parameters studied included the age and sex of the patients, the presence and type of symptoms, preoperative levels of serum calcium and parathyroid hormone (PTH), and the tumor weight of the parathyroid glands removed. Comparing two groups of such un- even sizes entails certain statistical precautions, which are discussed below.

\section{MATERIALS AND METHODS}

Over a 25-year period (June 1976 to December 2001), 311 consecutive patients (82 men and 229 women, aged $16-92$ years; mean $\pm \mathrm{SD}, 60.5 \pm 15.2$ years) underwent operation in the Department of Surgery of Geneva University Hospital for PHP: 242 had adenomas (78\%), 60 had hyperplasias (19\%), and 9 had PC (3\%). In the PC subgroup, there were seven women and two men, aged 35 to 67 years (mean $\pm \mathrm{SD}, 54.9 \pm 9.9$ years); none of them had a family history of PC. PC was defined by Schantz and Castelman's criteria, ${ }^{16}$ which include any of the following: (1) local invasion of contiguous structures; (2) nodal or distant metastases; and (3) capsular or vascular invasion, thick fibrous bands, pleiomorphic cells in a trabecular pattern, or mitoses (Fig. 1). 
TABLE 1. Characteristics of patients with parathyroid carcinoma $(n=9)$

\begin{tabular}{|c|c|c|c|c|c|c|c|c|}
\hline Patient no. & Sex/age (y) & Symptoms & $s-\mathrm{Ca}$ & $s-\mathrm{P}$ & $s$-PTH & OP & $\begin{array}{l}\text { Time of } \\
\text { diagnosis }\end{array}$ & Follow-up (y) \\
\hline 1 & $\mathrm{M} / 35$ & $\mathrm{U}, \mathrm{T}$, pancreatitis & 3.93 & $\downarrow$ & 19.4 & $\begin{array}{l}\mathrm{IR}+\mathrm{R} \text { thyroid } \\
\text { lobectomy }\end{array}$ & After OP & NED 25.2 \\
\hline 2 & $\mathrm{~F} / 62$ & $\mathrm{O}, \mathrm{U}, \mathrm{T}$ & 5.99 & 1.70 & 6.9 & IL & After OP & NED 21.3 \\
\hline 3 & $\mathrm{~F} / 51$ & $\mathrm{O}, \mathrm{U}$ & $2.4-2.8$ & $1.9-3.5$ & 12.8 & SR & During OP & $\begin{array}{l}\text { DOC } 3.0 \\
\text { (myocardial } \\
\text { infarct) }\end{array}$ \\
\hline 4 & $\mathrm{~F} / 57$ & $\mathrm{O}, \mathrm{U}, \mathrm{T}$ & 2.71 & $\downarrow$ & 4.6 & IR & After OP & NED 16.6 \\
\hline 5 & $\mathrm{~F} / 45$ & None & 3.60 & .40 & 4.1 & IL & During OP & $\begin{array}{l}\text { DOC } 5.0 \\
\text { (alcohol } \\
\text { abuse) }\end{array}$ \\
\hline 6 & $\mathrm{M} / 56$ & $\mathrm{O}, \mathrm{U}$ & 3.28 & .79 & 6.4 & $\begin{array}{l}\mathrm{SR}+\mathrm{R} \text { thyroid } \\
\text { lobectomy }+\mathrm{LN}\end{array}$ & During OP & NED 8.6 \\
\hline 7 & $\mathrm{~F} / 63$ & $\begin{array}{l}\mathrm{O}, \text { palpable } \\
\text { cervical mass }\end{array}$ & 3.27 & .66 & 20.0 & $\begin{array}{l}\mathrm{Lx} 2+\mathrm{L} \text { thyroid } \\
\text { lobectomy }\end{array}$ & During OP & NED 3.8 \\
\hline 8 & $\mathrm{~F} / 67$ & $\mathrm{~T}$ & 2.91 & .52 & 4.0 & $\begin{array}{l}\mathrm{IL}+\mathrm{L} \text { thyroid } \\
\text { lobectomy }\end{array}$ & After OP & NED 2.0 \\
\hline 9 & $\mathrm{~F} / 58$ & $\mathrm{O}, \mathrm{U}$ & 3.02 & .59 & 13.8 & $\begin{array}{l}\mathrm{IL}+\text { total } \\
\text { thyroidectomy }\end{array}$ & During OP & NED 1.3 \\
\hline
\end{tabular}

OP, operation; U, urinary symptoms; T, thymic disorders; IR, inferior right; R, right; NED, no evidence of disease; O, osteoarticular symptoms; IL, inferior left; SR, superior right; DOC, dead of other cause; LN, lymph node; L, left; s-Ca, calcium in serum; $s-P$, phosphorus in serum; $s$-PTH, parathyroid hormone in serum; Lx2, bilateral thyroid lobectomy (total thyroidectomy).

Reference values: $p$-Ca, 2.20 to $2.52 \mathrm{mmol} / \mathrm{L} ; p-\mathrm{P}, .80$ to $1.40 \mathrm{mmol} / \mathrm{L}$.

TABLE 2. Cases of parathyroid cancer ( $P C)$ compared with all cases of benign primary hyperparathyroidism (PHP) operated on during the same period

\begin{tabular}{|c|c|c|c|}
\hline Variable & $\mathrm{PC}$ & Benign PHP & $P$ value \\
\hline No. of cases & 9 & 302 & - \\
\hline Age $(y)$ & & & NS \\
\hline Mean \pm SD (range) & $54.9 \pm 9.9(35-67)$ & $60.7 \pm 15.4(16-92)$ & \\
\hline Median (IQR) & $57(11)$ & $62(21)$ & \\
\hline Sex, M/F (\% men $)$ & $2 / 7$ & $80 / 222(26 \%)$ & NS \\
\hline Patients with symptoms & 8 & $238(79 \%)$ & NS \\
\hline General symptoms & 4 & $68(23 \%)$ & NS \\
\hline Skeletal involvement & 5 & $84(28 \%)$ & $<.003$ \\
\hline Renal involvement & 5 & $84(28 \%)$ & NS \\
\hline Renal and skeletal involvement & 5 & $13(4 \%)$ & $<.0001$ \\
\hline Thymic disorders & 3 & $47(16 \%)$ & NS \\
\hline GI symptoms & 1 & $39(13 \%)$ & NS \\
\hline Hypertension & 0 & $52(13 \%)$ & NS \\
\hline$s-\mathrm{Ca}(\mathrm{mmol} / \mathrm{L})$ & & & .04 \\
\hline Mean \pm SD (range) & $3.4 \pm 1.1(2.6-6.0)$ & $2.9 \pm .3(2.0-4.8)$ & \\
\hline Median (IQR) & $3.3(.8)$ & $2.8(.4)$ & \\
\hline$s$-PTH ( $\times$ upper range of normal value) & & & $<10^{-5}$ \\
\hline Mean \pm SD (range) & $10.3 \pm 6.5(4.0-20.0)$ & $2.6 \pm 2.2(.5-24.1)$ & \\
\hline Median (IQR) & $6.9(9.3)$ & $2.0(1.5)$ & \\
\hline Tumor weight (g) & & & $<10^{-5}$ \\
\hline Mean \pm SD (range) & $4.9 \pm 2.1(1.9-8.0)$ & $1.3 \pm 2.2(.015-14.9)$ & \\
\hline Median (IQR) & $5.3(3.2)$ & $.6(1.0)$ & \\
\hline
\end{tabular}

NS, not significant; IQR, interquartile range; GI, gastrointestinal; s-Ca, calcium in serum; $s$-PTH, parathyroid hormone in serum. Continuous variables were compared by using the Mann-Whitney test, and proportions were compared by using Fisher's exact test. Symptoms are detailed in the text (see Materials and Methods). Reference values for $p$-Ca are 2.20 to $2.52 \mathrm{mmol} / \mathrm{L}$.

Symptoms were looked for in the entire PHP group and allocated to six possible categories: general (fatigue, weight loss, and anorexia), articular and/or skeletal (osteopenia or osteoporosis as measured by dualenergy x-ray absorptiometry, according to the World
Health Organization criteria ${ }^{17}$ ), renal (nephrolithiasis, nephrocalcinosis, or impaired renal function in the absence of any other etiology), gastrointestinal (anorexia, nausea, vomiting, dyspepsia, vague abdominal complaints, and constipation ${ }^{18}$ ), hypertensive, and 
TABLE 3. Weight of parathyroid glands and microscopic features of malignancy according to Schantz and Castleman ${ }^{16}$ found in nine patients with $P C$

\begin{tabular}{|c|c|c|c|c|c|}
\hline Patient no. & Sex/age (y) & $\begin{array}{l}\text { Weight of gland } \\
\text { removed }(\mathrm{g})\end{array}$ & $\begin{array}{l}\text { Capsular or vascular } \\
\text { invasion }\end{array}$ & $\begin{array}{c}\text { Thick fibrous } \\
\text { bands }\end{array}$ & $\begin{array}{c}\text { Pleiomorphic cells, trabecular } \\
\text { pattern, or mitoses }\end{array}$ \\
\hline 1 & $\mathrm{M} / 35$ & 6.0 & Vascular invasion & - & Trabecular pattern \\
\hline 3 & $\mathrm{~F} / 51$ & 5.0 & Vascular invasion & + & - \\
\hline 4 & $\mathrm{~F} / 57$ & 6.5 & Capsular invasion & + & - \\
\hline 5 & $\mathrm{~F} / 45$ & 8.0 & Capsular + vascular invasion & - & - \\
\hline 6 & $\mathrm{M} / 56$ & 4.0 & Capsular + vascular invasion & + & - \\
\hline 7 & $\mathrm{~F} / 63$ & NA & - & + & Mitoses \\
\hline 8 & $\mathrm{~F} / 67$ & 1.9 & Vascular invasion & + & Mitoses \\
\hline 9 & $\mathrm{~F} / 58$ & 5.7 & Capsular invasion & - & Trabecular pattern \\
\hline
\end{tabular}

PC, parathyroid carcinoma; NA, not available.

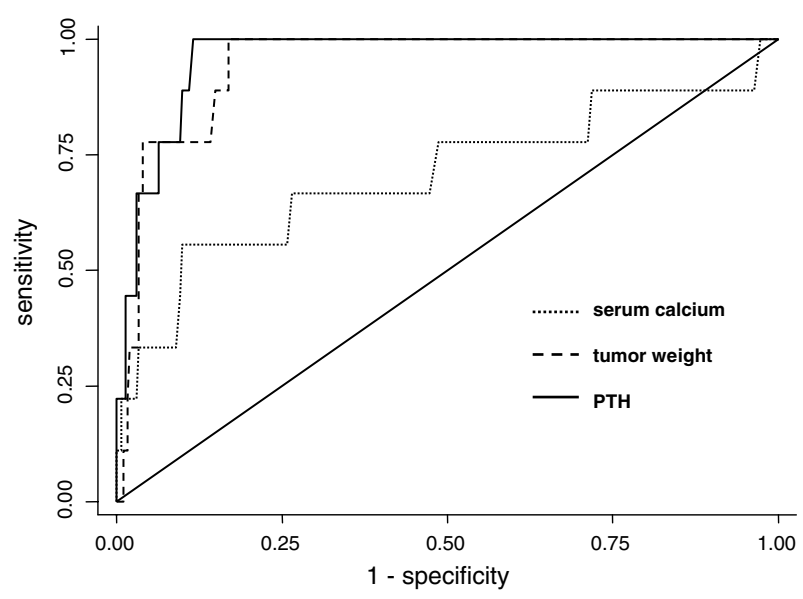

FIG. 2. Box plots for tumor weight and serum calcium and parathyroid hormone (PTH) determinations in the benign primary hyperparathyroidism (PHP) and carcinomatous cohorts. Tumor weights are provided in grams, serum calcium levels in millimoles per liter, and PTH values as multiples of the upper range of normal values.

thymic (or mood disorders). Starting in February 1986, all patients who underwent operation (5 with PC and 251 with benign PHP) received methylene blue in the hour preceding surgery to facilitate identification of the parathyroid gland involved. ${ }^{19}$

Summary results are given as mean $\pm \mathrm{SD}$. Comparisons between two groups for continuous variables were performed with a Mann-Whitney test. Fisher's exact test was used for comparing proportions.

Receiver operating characteristic curves were used to determine the performance of two serum parameters (calcium and PTH) and the weight of the parathyroid tumor removed to evaluate their ability to predict PC before surgery. Logistical regression was used to estimate the probability of diagnosing a PC based on serum calcium and PTH levels and the

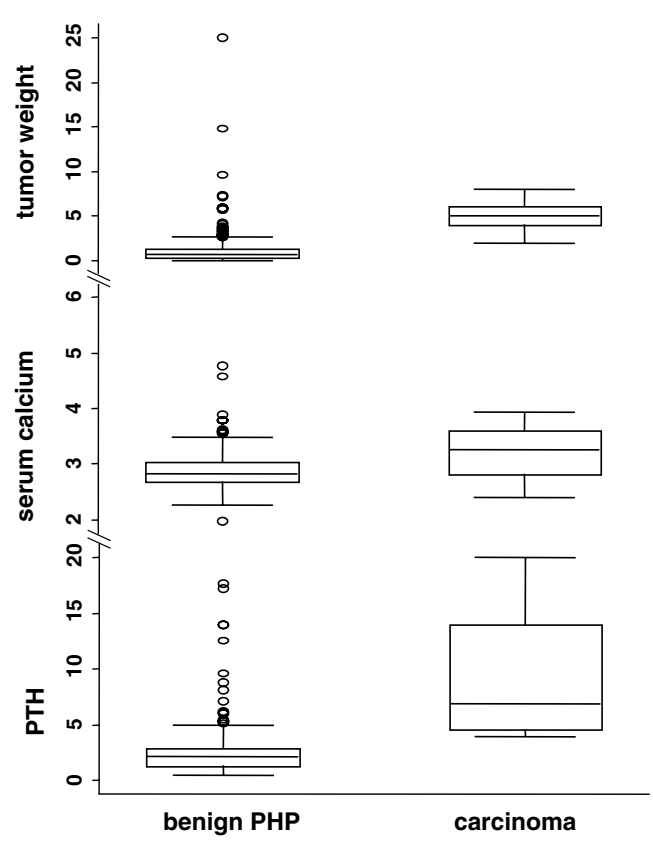

FIG. 3. Receiver operating characteristic (ROC) curves achieved by tumor weights, serum calcium, and parathyroid hormone (PTH) determinations. Areas under ROC curves measure .7051 for serum calcium determinations, .9446 for tumor weights, and .9601 for PTH determinations. PHP, primary hyperparathyroidism.

weight of the tumor. The effect of predictive variables was assessed by odds ratio (OR) and $95 \%$ confidence intervals. The significance levels for two-sided $P$ values was .05 in all tests. The data were analyzed with Stata software, release 7.0 (StataCorp LP, College Station, TX).

\section{RESULTS}

Characteristic features of patients with PC are listed in Table 1 and are compared in Table 2 with those 
TABLE 4. Ability of serum calcium, PTH levels, and tumor weight to differentiate PC from benigh PHP

\begin{tabular}{lcccr}
\hline Variable & Cutoff value & Sensitivity & Specificity & PPV \\
\hline Ca $(\mathrm{mmol} / \mathrm{L})$ & 3.27 & $56(21-86)$ & $90(76-93)$ & 14 \\
PTH (pmol/L or nmol/L) & $4 \times \mathrm{N}$ & $100(66-100)$ & $88(83-91)$ & 20 \\
Tumor weight $(\mathrm{g})$ & 1.9 & $100(66-100)$ & $81(76-86)$ & 15 \\
\hline
\end{tabular}

Except for cutoff values, all figures are percentages; numbers in parentheses are $95 \%$ confidence levels.

PTH, parathyroid hormone; PC, parathyroid carcinoma; PHP, primary hyperparathyroidism; PPV, positive predictive value; NPV, negative predictive value; $\mathrm{N}$, upper range of normal value.

TABLE 5. Odds ratio $(O R)$ estimate of parathyroid cancer compared with benign primary hyperparathyroidism

\begin{tabular}{lcccrr}
\hline Variable & Crude OR & $95 \%$ CI & $P$ value & Adjusted $^{\text {a }}$ OR & $95 \%$ CI \\
\hline Tumor weight & 1.28 & $1.07-1.52$ & .006 & .97 & $.78-1.19$ \\
Serum calcium & 5.51 & $1.86-16.35$ & .002 & 2.29 & $.73-7.18$ \\
PTH & 1.39 & $1.22-1.59$ & .000 & 1.37 & $1.16-1.61$ \\
\hline
\end{tabular}

CI, confidence interval; PTH, parathyroid hormone.

${ }^{a}$ Adjusted variables were tumor weight, serum calcium, and PTH; all variables were mutually adjusted.

of all patients who underwent operation for benign PHP during the same period. Eight of the nine patients with PC had symptoms, versus 238 of 302 in the benign PHP group (not significant). Compared with patients who had adenomas and hyperplasias, patients with PC were more likely to have bone disease $(P<.003)$ or concomitant bone and renal disease $(P$ $<$.0001). However, for a given level of increased $\mathrm{PTH}$, symptoms as a whole were evenly distributed whether patients had PC or benign PHP. Within the PC subgroup, one patient presented with a palpable neck mass, but none had preoperative (or postoperative) vocal cord paralysis, and none had regional (lymph nodes) or distant metastases at the time of diagnosis.

The nine patients with PC had their pathologic gland removed, and frozen sections were performed. The gland removed was described as firm in one case, dark in another, and adherent or "in continuity" with the thyroid (in three) or the esophagus (in one). Microscopic criteria for malignancy according to Schantz and Castleman ${ }^{16}$ are listed in Table 3. Carcinomatous glands were significantly bigger than adenomatous and hyperplastic glands $(4.9 \pm 2.1 \mathrm{~g}$ vs. $1.3 \pm 2.2 \mathrm{~g}$, respectively; $P<.00001$ ).

Frozen sections were unable to prove cancer in four patients. Excision of the thyroid lobe adjacent to PC was performed in five patients (three times in a second step upon receipt of the definite diagnosis) because definite slides had shown the surgical section to be invaded. In none of these three cases, however, did histology confirm invasion of the thyroid tissue removed. In two instances, thyroidectomy was added because of intrinsic benign thyroid pathologies, irrespective of PC. In addition to thyroid lobectomy, one patient had benign-looking internal jugular lymph nodes removed, and these also proved benign. No adjuvant treatment was proposed for any of the PC patients.

Two patients with PC died of unrelated causes 3 and 5 years after surgery. The remaining seven patients with PC are alive after a median follow-up period of 8 years (range, 3 months to 20 years), and yearly serum calcium determinations are so far within the normal range.

The ability of increased PTH levels and tumor weights to predict PC was considerably greater than that of hypercalcemia (Figs. 2 and 3 and Table 4), even though serum calcium levels were also higher in the PC subgroup (Table 2). For the four patients whose cancers were undiagnosed during surgery, both PTH levels and tumor weights exceeded respective cutoff values.

To evaluate the probability of diagnosing PC, a logistic regression method was used that was based on serum calcium and PTH levels and the weight of the tumor removed (Table 5). Crude OR showed that patients with higher levels of serum calcium and PTH and heavier tumor weights were more likely to have PC. After adjusting for tumor weight and serum calcium, the OR associated with PTH did not change, whereas the discriminating effect of tumor weight and calcium both disappeared (this means that all discriminative information can be derived from PTH levels alone). The $R^{2}$ value was .26, and this means that approximately one fourth of the variance was explained by these three factors. 


\section{DISCUSSION}

Several factors account for the difficulty in establishing a correct diagnosis of PC. First, PC mimics benign PHP clinically, and given the rarity of PC (it never exceeds $5 \%$ of cases of benign PHP in series of respectable sizes: $2.9 \%$ in ours but $1 \%$ in most series $^{9,11,12,20}$ ), the diagnosis is seldom suspected. Second, although parathyroid infiltration into adjacent neck structures is obviously synonymous with PC, ${ }^{3,4,15,21}$ such extreme conditions are not the rule: only 38 cases $(28 \%)$ in a 1991 Japanese survey of 138 cases $^{20}$ (however, some degree of adherence to the thyroid gland is quite regularly observed ${ }^{22}$-four times in the present series).

Thus, the scarcity in PC of macroscopic signs customarily suggestive of neoplasia (as in this series) may account for changes in the histological criteria of the disease, because most authors ${ }^{18}$ have now adopted Schantz and Castleman's ${ }^{16}$ definition, by which more discrete histological features suffice in establishing a diagnosis of PC. ${ }^{1,3}$ These features (which have been used in this study) include possible combinations of the following: a trabecular pattern, mitotic figures, thick fibrous bands, or capsular and blood vessel invasion. For obvious reasons, diagnosis is even more difficult in the frozen-section setting ${ }^{8}$ (incorrect in four of our nine cases) and may lead to parsimonious tumor resections in conditions when more generous ones (if not necessarily more curative) would seem safer.

Curiously enough, PC frequently displays other features that may facilitate its diagnosis before surgery. Female prevalence is not as striking as in PHP, ${ }^{10,14,16,20,23,24}$ and patients tend to be younger, even though neither sex nor age reached statistical significance in this report. ${ }^{9}$ All but one of our nine patients were clearly symptomatic; the most discriminative finding in our series was a combination of urinary and skeletal symptoms ${ }^{10,14,22}$ (it is indeed a classic feature of PC to be more frequently and more severely symptomatic compared with benign $\mathrm{PHP}^{1,18}$ ). A palpable neck mass, ${ }^{6,8,10}$ described in nearly 1 in 3 cases of PC in a series of $61,{ }^{16}$ was noted only once in this study.

The severity of hypercalcemia may prove more useful than symptoms for suggesting PC before surgery: whereas the 302 patients with benign PHP had calcemias averaging $2.89 \pm .33 \mathrm{mmol} / \mathrm{L}$, for the 9 patients with PC, levels increased to $3.45 \pm 1.05$ $\mathrm{mmol} / \mathrm{L}$ (a significant difference; $P<.04$ ). Such differences have already been reported ${ }^{9}$; several series have shown $65 \%^{20}$ to $75 \%^{4}$ of $\mathrm{PC}$ patients with calcemias $>3.5 \mathrm{mmol} / \mathrm{L}$. Despite such differences, the sensitivity and, particularly, the positive predictive value of serum calcium for $\mathrm{PC}$ are disappointing (56\% and $14 \%$, respectively), as reflected by a considerable overlapping of patients with $\mathrm{PC}$ and benign PHP (Fig. 2). Increments are more striking for mean PTH determinations ${ }^{18,20}: 2.6$ times the upper level of normal value in cases of benign PHP, versus 10.3 in PC $\left(P<10^{-5}\right)$. This was confirmed in a series of 43 cases from the Mayo Clinic (mean increase, 10.2 times the upper limit of normal) ${ }^{14}$ but was not the case in a smaller California report, which failed to disclose significant differences in PTH levels between adenomas and PC. ${ }^{22}$ To a slightly lesser degree, the same holds true for tumor weight, a finding clearly demonstrated in an American series in which 10 PC patients had parathyroid glands exceeding $2.5 \mathrm{~g}$ (mean, $7.2 \mathrm{~g}$ ). ${ }^{6}$ Although more promising than serum calcium, PTH increments and tumor weight are unable to predict PC reliably in all instances (indeed, when the prevalence of a disease is low, false-positive results can be expected to outnumber true-positive ones: even when the sensitivity and specificity of a given test are high, the positive predictive value is still low). However, the probability of PC was nil in our series when the PTH was increased $<4$ times the upper limit of normal, the tumor weight was $<1.9 \mathrm{~g}$, or both.

The most effective treatment in PC is surgical and should consist of an en-bloc resection, avoiding rupture and spillage of the tumor. ${ }^{20}$ In the event of a correct diagnosis of PC being available during surgery (and, obviously, also before), attention should be given to the possible invasion of the resection margins. Doubt on the thyroid side (or the presence of nodular thyroid disease) should entail en-bloc ipsilateral thyroidectomy ${ }^{13}$ (even though histology may fail to find residual cancer in the thyroid specimen resected, as occurred four times in this series). By the same token, jugular lymph node dissection should be performed only in cases of grossly enlarged lymph nodes, a situation reported 9 times in 18 patients with metastatic disease in a series of $59^{16}$ : we performed it once in a patient with normal-appearing nodes, but histology failed to disclose lymphatic metastases. This rather aggressive attitude seems justified by the futility of repeat surgery $y^{22,25}$ or of other more conservative treatments in case of recurrent PC. $2,6,11-14$

In conclusion, there are intraoperative indications clearly suggestive of $\mathrm{PC}$, such as gland firmness or infiltration into adjacent structures, usually the thyroid. These findings, however, may be absent, as in 
this series. Consequently, one should seriously contemplate the diagnosis of $\mathrm{PC}$ in the presence of an abnormally large parathyroid gland and/or of unusually high levels of serum calcium or-better still - of PTH, even if this study failed to pinpoint irrefutable and pathognomonic cutoff values for these three parameters to assess PC. However, the diagnosis of PC can be reliably ruled out for the many patients in whom PTH increments are inferior to 4 times the upper limit of normal range, tumor weights are $<1.9$ $\mathrm{g}$, or both, and this should help to reassure the surgeon who renounces extensive resections, because the probability of PC is nil below these values.

\section{REFERENCES}

1. Obara T, Okamoto T, Kanbe M, Iihara M. Functioning parathyroid carcinoma: clinicopathologic features and rational treatment. Semin Surg Oncol 1997;13:134 41.

2. Fujimoto Y, Obara T, Ito Y, Kodama T, Nobori M, Ebihara S. Localization and surgical resection of metastatic parathyroid carcinoma. World J Surg 1986;10:539-47.

3. Smith JF, Coombs RRH. Histological diagnosis of carcinoma of the parathyroid gland. J Clin Pathol 1984;37:1370-8.

4. Holmes EC, Morton DL, Ketcham AS. Parathyroid carcinoma: a collective review. Ann Surg 1969;169:631-40.

5. Sloan DA, Schwartz RW, McGrath PC, Kenady DE. Diagnosis and management of thyroid and parathyroid hyperplasia and neoplasia. Curr Opin Oncol 1995;7:47-55.

6. van Heerden JA, Weiland LH, ReMine WH, Walls JT, Purnell DC. Cancer of the parathyroid glands. Arch Surg 1979;114: 475-80

7. Fujimoto Y, Obara T. How to recognize and treat parathyroid carcinoma. Surg Clin North Am 1987;67:343-57.

8. Sandelin K, Auer G, Bondeson L, Grimelius L, Farnebo LO. Prognostic factors in parathyroid cancer: a review of 95 cases. World J Surg 1992;16:724-31.

9. Palnaes HC, Lau PM, Christensen L. Diagnosis, treatment and outcome of parathyroid cancer: report of eight patients. Eur $J$ Surg 1991;157:517-20.
10. Wang C, Gaz RD. Natural history of parathyroid carcinoma. Diagnosis, treatment, and results. Am J Surg 1985;149:522-7.

11. Shane E. Parathyroid carcinoma. J Clin Endocrinol Metab 2001;86:485-93.

12. Trigonis C, Cedermark B, Willems J, Hamberger B, Granberg PO. Parathyroid carcinoma: problems in diagnosis and treatment. Clin Oncol 1984;10:11-9.

13. Shortell CK, Andrus CH, Phillips CE, Schwartz SI. Carcinoma of the parathyroid gland: a 30 year experience. Surgery 1991;110:704-8.

14. Wynne AG, van Heerden J, Carney JA, Fitzpatrick LA. Parathyroid carcinoma: clinical and pathologic features in 43 patients. Medicine 1992;71:197-205.

15. Anderson BJ, Samaan NA, Vassilopoulou-Sellin R, Ordonez NG, Hickey RC. Parathyroid carcinoma: features and difficulties in diagnosis and management. Surgery 1983;94:906-15.

16. Schantz A, Castleman B. Parathyroid carcinoma. A study of 70 cases. Cancer 1973;31:600-5.

17. Kanis JA, Melton LJ, Christiansen C, Johnston CC, Khaltaev N. Perspective: the diagnosis of osteoporosis. $J$ Bone Miner Res 1994;9:1137-41.

18. Shane E, Bilezikian JP. Parathyroid carcinoma: a review of 62 patients. Endocr Rev 1982;3:218-26.

19. Robert J, Delay D, Bühler L, Garcia A, Ludwig C, Spiliopoulos A. Intérêt du bleu de méthylène dans la chirurgie de l'hyperparathyroïdisme primaire. Ann Endocrinol (Paris) 1998;59:23-6.

20. Obara T, Fujimoto Y. Diagnosis and treatment of patients with parathyroid carcinoma: an update and review. World J Surg 1991;15:738-44.

21. Bondeson L, Sandelin K, Grimelius L. Histopathological variables and DNA cytometry in parathyroid carcinoma. Am J Surg Pathol 1993;17:820-9.

22. Levin KE, Galante M, Clark OH. Parathyroid carcinoma versus parathyroid adenoma in patients with profound hypercalcemia. Surgery 1987;101:649-60.

23. Hundahl SA, Fleming ID, Fremgen AM, Menck HR. Two hundred eighty-six cases of parathyroid carcinoma treated in the US between 1985-1995. A National Cancer Data Base report. Cancer 1999;86:538-44.

24. Mundy GR, Cove DH, Fisken R. Primary hyperparathyroidism: changes in the pattern of clinical presentation. Lancet 1980;1:1317-20.

25. Kebebew E, Arici C, Duh QY, Clark OH. Localization and reoperation results for persistent and recurrent parathyroid carcinoma. Arch Surg 2001;136:878-85. 